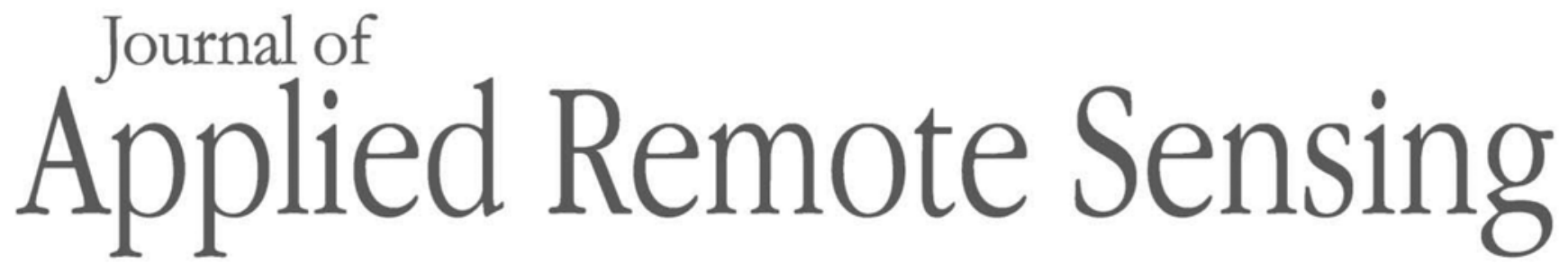

RemoteSensing.SPIEDigitalLibrary.org

\title{
Improving the image fusion procedure for high-spatiotemporal aerosol optical depth retrieval: a case study of urban area in Taiwan
}

\author{
Chih-Yuan Huang \\ Hsuan-Chi Ho \\ Tang-Huang Lin
}




\title{
Improving the image fusion procedure for high-spatiotemporal aerosol optical depth retrieval: a case study of urban area in Taiwan
}

\author{
Chih-Yuan Huang, ${ }^{\text {a,b }}$ Hsuan-Chi Ho, ${ }^{\text {b }}$ and Tang-Huang Lin ${ }^{\text {a,* }}$ \\ ${ }^{a}$ National Central University, Center for Space and Remote Sensing Research, \\ Taoyuan City, Zhongli District, Taiwan \\ ${ }^{\mathrm{b}}$ National Central University, Department of Civil Engineering, Taoyuan City, \\ Zhongli District, Taiwan
}

\begin{abstract}
Numerous studies employed remote sensing techniques on regional air quality in terms of aerosols, in particular, the observations from polar-orbiting satellites offer more detail on spatial distribution. Since the air pollutants/aerosols dramatically vary in location with time, diurnal observations on a timescale are restricted by the temporal resolution of the polar-orbiting satellite. To address this issue, this research proposes a spatially and temporally adaptive reflectance fusion model for measuring atmospheric properties to synthesize high-spatial-temporal resolution images from polar and geostationary satellite imagery for air quality monitoring. The reflectivity from short-wave infrared is employed to preserve the atmospheric effect within the fused image in the green band for further aerosol optical depth (AOD) retrieval. Taking the Landsat- 8 Operational Land Imager as the reference, the spatial resolution of the Himawari- 8 Advanced Himawari Imager (in kilometers) can thus be resampled into $30 \mathrm{~m}$ every $10 \mathrm{~min}$ during the daytime, by considering the surface bidirectional reflectivity from the variation of the solar zenith angle. The AOD retrieved with fused images containing atmospheric effect could have a better performance after comparison with in situ measurements, and therefore, be suggested for high-spatial-temporal aerosol monitoring. () The Authors. Published by SPIE under a Creative Commons Attribution 3.0 Unported License. Distribution or reproduction of this work in whole or in part requires full attribution of the original publication, including its DOI. [DOI: 10 .1117/1.JRS.12.042605]
\end{abstract}

Keywords: high-spatial-temporal image fusion; atmospheric effect; short-wave infrared spectrum; Himawari-8 Advanced Himawari Imager; Landsat-8 Operational Land Imager; aerosol optical depth.

Paper 180372SS received May 3, 2018; accepted for publication Jul. 25, 2018; published online Sep. 4, 2018.

\section{Introduction}

Remote sensing imaging from satellites is widely applied to environmental monitoring, such as land cover analysis, ${ }^{1,2}$ weather analysis, ${ }^{3}$ and crop phenology monitoring. ${ }^{4}$ Among these applications, some of them require better spatial and temporal resolutions, such as disaster management, crop phenology monitoring, land cover change detection, and air quality monitoring. ${ }^{5}$ Recently, with urban development and industrialization, air pollution has become an important issue that endangers human health ${ }^{6-8}$ and has a negative effect on the earth energy budget. Thus, the monitoring of air pollutants is essential to understand and eliminate the effect and threat of suspended particles (atmospheric aerosols). Ground stations are widely used to monitor the air quality in particulate matter (PM) concentrations near the surface. Although they can examine air quality effectively, the spatial resolution of ground stations is usually too coarse to support local phenomena. ${ }^{9}$ With the advantage of wide coverage in regional or global scales, satellite observation becomes a better choice, with higher spatial information on air quality monitoring in

*Address all correspondence to: Tang-Huang Lin, E-mail: thlin@csrsr.ncu.edu.tw 
aerosol optical properties..$^{5,10,11}$ The aerosol optical depth (AOD) can be retrieved from satellite images to further estimate $\mathrm{PM}$ concentrations such as $\mathrm{PM}_{2.5}$ and $\mathrm{PM}_{10} \cdot{ }^{12,13}$

In general, the regional emission and transportation of pollutants result in the air quality change, both dynamically and locally. Thus, the spatial and temporal resolutions of a single polar-orbiting satellite are insufficient to examine the diurnal variation of air quality. As we all know, no single satellite can produce images with both high-spatial and high-temporal resolutions. Although WorldView-3 (0.3 to $1.3 \mathrm{~m}$ ) and Landsat-8 (15 to $30 \mathrm{~m})$ have relatively highspatial resolutions, their temporal resolutions are low, which limits continuous monitoring. On the other hand, satellites with high-temporal resolution can be applied for time-critical applications, such as Brakenridge and Anderson ${ }^{14}$ applied terra/aqua moderate-resolution imaging spectroradiometer (MODIS, 1 to 2 days) images on disaster management, and Imai and Yoshida ${ }^{15}$ applied Himawari-8 Advanced Himawari Imager (AHI, $10 \mathrm{~min}$ ) for weather monitoring. However, the coarse spatial resolutions cannot provide detailed information in the spatial sphere. Hence, image fusion techniques were proposed to generate high-spatial-temporal resolution imagery, ${ }^{16-20}$ such as the image-fusion model for enhancing the temporal resolution of Landsat- 8 surface reflectance images using MODIS and further application to surface temperature monitoring. ${ }^{21,22}$

Himawari-8, the second generation Asian geostationary satellite launched in 2014, offering observations every $10 \mathrm{~min}$, has been widely applied to the investigations of short-term phenomena in dynamic procedures even with its low spatial resolution. ${ }^{3,4,15}$ In addition to a hightemporal resolution, the new payload named the AHI equips with 16 bands within the visible and infrared spectra and facilitates detailed information on both terrestrial and atmospheric parameters. The similarity of the visible bands between Landsat- 8 Operational Land Imager (OLI) and Himawari-8 AHI sensors, as shown in Table 1, offers a high potential to fuse the high-spatial resolution $(30 \mathrm{~m})$ with the ultrahigh-temporal resolution (10-min windows) imagery for the monitoring of short-term events. Therefore, this research aims at applying a spatialtemporal image fusion method on Landsat- 8 and Himawari- 8 images for the further application of air quality monitoring. To achieve this objective, there are two major challenges that need to be addressed.

(1) Atmospheric effect. The existing image fusion approaches are generally based on the surface reflectance for land cover applications. If we directly apply top-of-atmosphere (TOA) reflectance in the existing image fusion methods, areas that have large changes caused by the atmospheric scattering/absorption effect would be treated as land cover changes and discarded in the fusion process. For research that focuses on retrieving atmospheric phenomena from the fused imagery, we need to modify the existing methods to preserve the atmospheric properties within the fused images.

Table 1 The specifications of Himawari-8 AHI and Landsat-8 OLI.

\begin{tabular}{|c|c|c|c|c|}
\hline \multirow[b]{2}{*}{ Satellite/sensor } & \multicolumn{2}{|c|}{ Himawari-8/AHI } & \multicolumn{2}{|c|}{ Landsat-8/OLI } \\
\hline & \multicolumn{2}{|c|}{ Geostationary } & \multicolumn{2}{|r|}{ Polar } \\
\hline Orbit & \multicolumn{2}{|r|}{35,793} & \multicolumn{2}{|r|}{705} \\
\hline Altitude (km) & Band no. & Central wavelength $(\mathrm{nm})$ & Band no. & Central wavelength $(\mathrm{nm})$ \\
\hline \multirow[t]{6}{*}{ Spectral band } & 1 & 470.6 & 1 & 482.5 \\
\hline & 2 & 510.0 & 2 & 561.5 \\
\hline & 3 & 639.1 & 3 & 654.5 \\
\hline & 4 & 856.7 & 4 & 865.0 \\
\hline & 5 & 1610.1 & 5 & 1608.5 \\
\hline & 6 & 2256.8 & 6 & 2200.5 \\
\hline
\end{tabular}




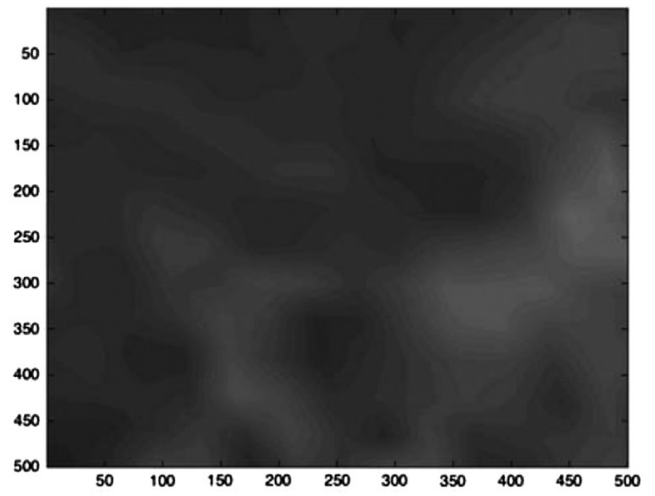

(a)

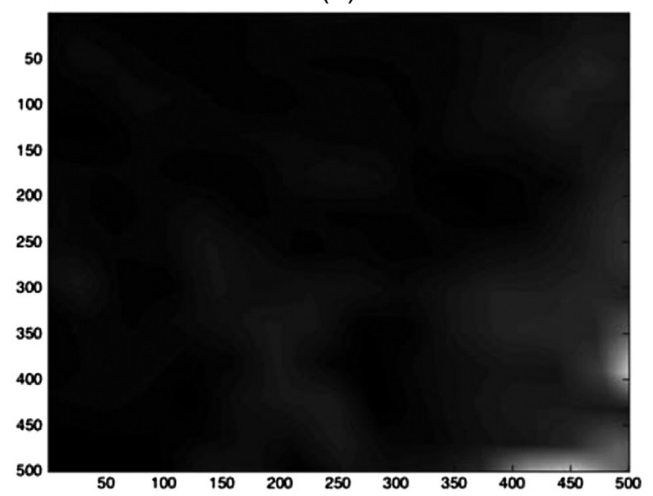

(c)

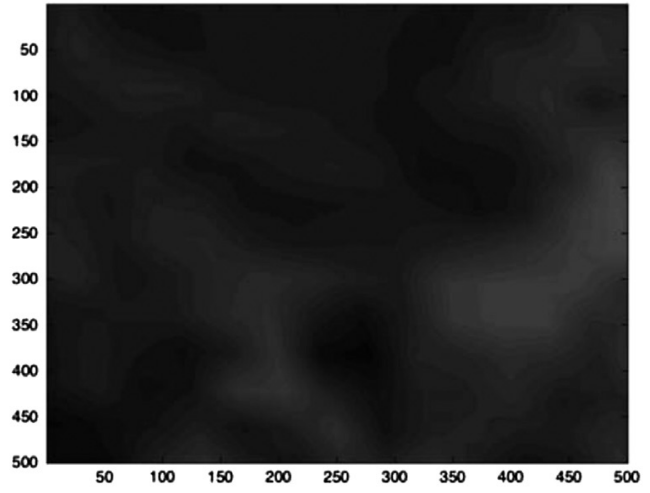

(b)

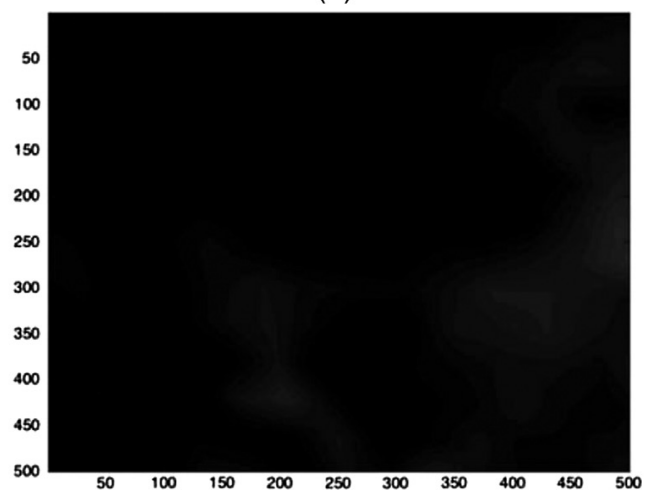

(d)

Fig. 1 The hourly Himawari-8 satellite images of TOA reflectance in the green band at (a) $0900 \mathrm{~h}$, (b) 1100 h, (c) 1300 h, and (d) 1400 h during daytime on November 16, 2015.

(2) Bidirectional reflectivity. As much of the related work usually chose Landsat and MODIS images as their testing datasets, these two polar-orbiting satellites have similar local times of observation (solar zenith angle/incidence), which results in similar surface and TOA reflectance. However, as the Himawari-8 satellite produces images every $10 \mathrm{~min}$, there are significant reflectivity variations with the solar zenith angle between the images taken during a daytime. Examples of the variance in TOA reflectance with the solar zenith are shown in Fig. 1. These changes in surface reflectance also cause problems in image fusion as existing methods assumed that the reflectance does not change significantly across time.

Overall, three main objectives are proposed in this study. First, we aim at examining the feasibility of fusing Himawari-8 and Landsat- 8 satellite images to produce sequential images with ultrahigh-temporal resolution (10-min windows) and high-spatial resolution $(30 \mathrm{~m})$. The second objective is to modify the spatial and temporal adaptive reflectance fusion model (STARFM) algorithm ${ }^{16}$ for preserving the atmospheric information after image fusion. Finally, the fused images will be applied to aerosol retrieval for monitoring the air quality/pollution in highly spatiotemporal resolutions. To verify the results of fused images in aerosol retrieval, the in situ measurements of aerosol property from the aerosol robotic network (AERONET) are compared.

For the rest of this paper, we first review the literature related to spatial and temporal image fusion methods in Sec. 2. Section 3 introduces the details of the proposed spatial and temporal adaptive reflectance fusion model-aerosol property (STARFM-AP) methods and workflow. In Sec. 4, we explain the evaluation results by comparing them with an observed/actual Landsat image and the AOD observations from an AERONET ground station. The overall result is discussed in Sec. 5, and the conclusions and future work are followed up in Sec. 6. 


\section{Literature Review}

The function of the image fusion approach is to produce a better resolution in the spatial and temporal scales from multiple sources of satellite images. There are two general types of function. The first one is the spectral and spatial image fusion, which integrates multispectral images (e.g., RGB images) and panchromatic images to enhance the spatial resolution of multispectral images. ${ }^{23}$ The second type is the spatial and temporal image fusion, which aims at integrating high-spatial resolution imagery with high-temporal resolution imagery, such as STARFM. ${ }^{16}$

The main concept of STARFM is to estimate the new surface reflectance at a prediction date based on the reflectance differences between the Landsat and MODIS images at a reference date (i.e., the reference image). As a MODIS pixel may contain multiple land cover types (mixel), the STARFM predicts a new reflectance based only on the information from the neighborhood pixels with the same land cover type. Although the STARFM can be effective for regions that do not have significant land cover changes, the STARFM is less effective if the land cover changed. Thus, there are some efforts on fixing this issue, such as the spatial-temporal adaptive algorithm for mapping reflectance change (STAARCH) ${ }^{17}$ and the enhanced spatial and temporal adaptive reflectance fusion model. ${ }^{18}$ The idea of STAARCH is to find the changed area from reference image-pairs, and then, to use a series of MODIS images to identify the date of the change for the image fusion process. On the other hand, the ESARFM further assumes that the reflectance is linearly changed over time, thus, interpolating or extrapolating the variation of reflectance for fusing an image.

In addition to the STARFM community, another spatial and temporal image fusion group focuses on the spatial and temporal data fusion model (STDFM).${ }^{19}$ The STDFM first collects the surface reflectance differences between fine-resolution and coarse-resolution images and then calculates the mean surface reflectance of each land cover type based on an assumed relationship between the reflectance of the coarse and fine images. However, as using the mean reflectance for pixels of each land cover type may induce some uncertainties, the enhanced spatial and temporal data fusion model (ESTDFM) ${ }^{20}$ applies a sliding window to calculate the mean surface reflectance of each land cover type inside that window. Therefore, the ESTDFM can adaptively adjust the reflectance of the fused image according to local information.

However, the existing methods are not suitable for the objective of AOD retrieval because of the following factors. First, the fused images should employ the TOA reflectance to keep atmospheric information, where all the existing methods can only fuse the surface reflectance as they would punish the pixels that have large reflectance changes by reducing their contribution to the fusion process. The reflectance changes can be caused by the atmosphere, making it important information that needs to be retained. Second, as the air quality usually has dynamic and nonlinear changes, even for methods that consider large reflectance changes such as the ESTDFM and the STAARCH, their assumptions of the reflectance change behavior are not suitable. Hence, this study aims at extending the STARFM method to solve the above-mentioned issues for the objective of aerosol retrieval.

\section{Materials and Methodology}

The satellite images and in situ measurements of AOD on the AERONET NCU_Taiwan site $(24.968 \mathrm{~N}, 121.188 \mathrm{E})$ are collected for examining the proposed approach, including three dates of Landsat OLI, nine dates of Himawari-8 AHI and AOD measurements, as indicated in Table 2. The total number of tested images is 208. The reference images of the Landsat8 OLI and Himawari-8 AHI are selected on November 16, 2015 at 1020 h. They are marked with the red rectangular boxes on the left-hand side in Fig. 5. The Landsat-8 OLI images on September 13, 2015 and October 15, 2015 are employed as the observed images to compare with the fused images of Himawari-8 AHI; the results are shown in Figs. 6 and 7, respectively. The AERONET is a global aerosol observation network of Cimel Sun photometers. ${ }^{24}$ Since AERONET can provide high-quality aerosol properties, the AOD measurement of AERONET becomes a standard reference for validation of aerosol retrievals and further application. ${ }^{25}$ Thus the AOD measurements of AERONET NCU_Taiwan site located in the study area are collected for the reference AOD in this study. 
Huang, Ho, and Lin: Improving the image fusion procedure for high-spatiotemporal aerosol optical depth...

Table 2 The study area and testing datasets.

\begin{tabular}{|c|c|c|}
\hline Study area & Zhongli district & n City, Taiwan \\
\hline Reference AOD & Sun photometer meas & JET NCU_Taiwan site) \\
\hline & Landsat-8 & Himawari-8 \\
\hline Satellite image & Date (number of images) & Date (number of images) \\
\hline & September 13, 2015 (1) & September 13, 2015 (1) \\
\hline & October 15, 2015 (1) & October 15, 2015 (1) \\
\hline & November 16, 2015 (1) & October 25, 2015 (32) \\
\hline & & October 27, 2015 (32) \\
\hline & & November 15, 2015 (29) \\
\hline & & November 16, 2015 (32) \\
\hline & & November 27, 2015 (30) \\
\hline & & December 13, 2015 (22) \\
\hline & & December 19, 2015 (30) \\
\hline
\end{tabular}

In this study, there are three main stages proposed in the workflow including the preprocessing stage, the spatiotemporal image fusion stage, and the validation stage as shown in Fig. 2. Geometric registration, land cover type classification, and bidirectional reflectance correction of imagery data are implemented in the preprocessing stage. The second stage then performs the spatial and temporal image fusion process to predict the high-spatial resolution image at $T_{1}$ (i.e., synthetic image). Finally, the third stage retrieves AODs from the fused images and compares them with the in situ observations from the AERONET.

\subsection{Preprocessing of Landsat-8 and Himawari-8 Data}

The closest observation time between the Landsat- 8 and Himawari- 8 AHI images are first collected as the reference time $T_{\mathrm{R}}$ (i.e., Lansat $T_{\mathrm{R}}$ and $\mathrm{AHI}_{T_{\mathrm{R}}}$ ) for the fusing target of Himawari-8 images at the processing time at $T_{1}$ (i.e., $\mathrm{AHI}_{T_{1}}$ ). The preprocessing stage contains the geometric

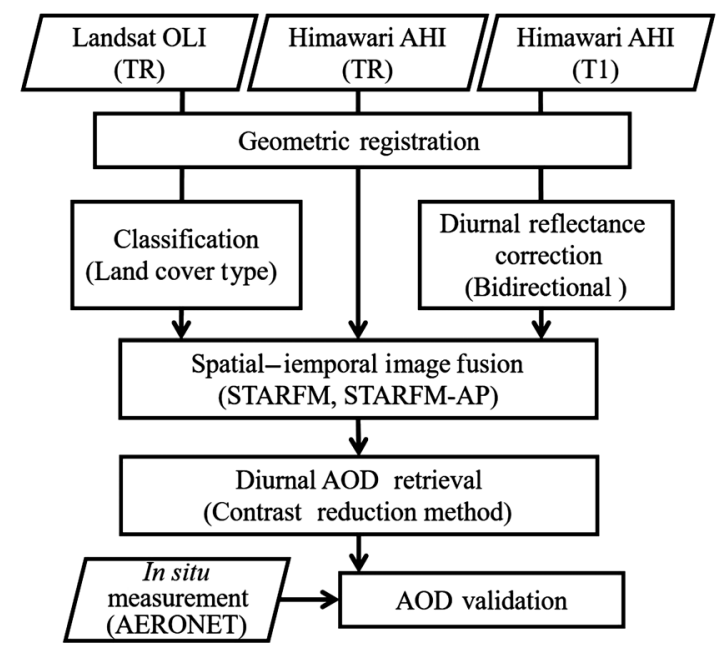

Fig. 2 The overall workflow of this study. 
registration of the images, the classification of the Lansat $_{T_{\mathrm{R}}}$, and the surface bidirectional reflectance correction of the $\mathrm{AHI}_{T_{1}}$.

Before the fusion process, the images are first geographically registered so that the information on the images can match with each other. In this study, we manually select tie points on the images and then apply affine transformation for the image registration. This simplified approach is selected because polar-orbiting satellites usually has a small field of view, which results in linear geometric distortion behavior and can usually be corrected by applying affine transformation. In addition, this stage also performs image classification on the Lansat $T_{\mathrm{R}}$ image to select spectrally similar pixels during the image fusion process. In this study, the $K$-means algorithm is used for land cover type classification.

Moreover, as mentioned earlier, the Himawari- 8 images are taken at different times during the daytime, which result in differences of the surface reflectivity. These differences will be mistaken as noise or land cover changes in the image fusion processing. Thus the bidirectional reflectance correction of the $\mathrm{AHI}_{T_{1}}$ image is essential in the preprocessing stage. In this study, we apply diurnal histogram matching to register the surface reflectance of the $\mathrm{AHI}_{T_{1}}$ image to that of the $\mathrm{AHI}_{T_{\mathrm{R}}}$ image.

\subsection{Spatial-Temporal Image Fusion Stage}

Two spatial and temporal image fusion methods are examined in this study. One is the original STARFM model ${ }^{16}$ and STARFM-AP proposed for atmospheric properties, as briefly introduced in Secs. 3.2.1 and 3.2.2, respectively.

\subsubsection{STARFM model}

Assume that the surface reflectance differences between the fine-resolution and coarse-resolution images at the reference time equal the differences at the prediction time $T_{\mathrm{R}}$. The surface reflectance of a specific pixel at $T_{1}$ can be estimated by ${ }^{16}$

$$
F\left(x_{i}, y_{j}, t_{1}\right)=C\left(x_{i}, y_{j}, t_{1}\right)+F\left(x_{i}, y_{j}, t_{R}\right)-C\left(x_{i}, y_{j}, t_{R}\right),
$$

where the $x_{i}, y_{j}$ identify an image pixel and the $F$ and $C$ represent the fine-resolution and coarseresolution images, respectively. Considering the effects of multiple land cover types within a single pixel in coarse resolution, the STARFM includes more information from the spectrally similar neighboring pixels. Thus Eq. (1) is modified into ${ }^{16}$

$$
F\left(x_{w / 2}, y_{w / 2}, t_{1}\right)=\sum_{i}^{w} \sum_{j}^{w} W_{i j} \times\left[C\left(x_{i}, y_{j}, t_{1}\right)+F\left(x_{i}, y_{j}, t_{R}\right)-C\left(x_{i}, y_{j}, t_{R}\right)\right],
$$

where the $w$ is the window size for finding spectrally similar neighboring pixels, the $W_{i j}$ is the combined weighting of a neighboring pixel including the spectral, temporal, and distance factors. In general, if the surface reflectance difference between the Lansat $T_{\mathrm{R}}$ and $\mathrm{AHI}_{T_{\mathrm{R}}}$ is large, the spectral weighting is lowered. If a pixel has a large reflectance difference between the $\mathrm{AHI}_{T_{\mathrm{R}}}$ and the $\mathrm{AHI}_{T_{1}}$, the temporal weighting is lowered. In addition, a neighboring pixel that is spatially close to the central pixel has a higher distance weighting than a neighboring pixel that is distant from the central pixel. It is noted that Eq. (2) is revised from the original STARFM equation for only one pair of reference images. Finally, a high-spatial resolution image at $T_{1}$ can be generated by going through every pixel. Refer to Gao et al. ${ }^{16}$ for more details on the STARFM model.

\subsubsection{STARFM-AP approach}

To keep the atmospheric information, the TOA reflectance from the coarse image (Himawari-8 $\mathrm{AHI}_{T_{1}}$ image) is utilized instead of the surface reflectance to produce the fine-spatial resolution image at $T_{1}$. However, if we directly apply the TOA reflectance from the Landsat $\mathrm{OLI}_{T_{\mathrm{R}}}$ and Himawari $\mathrm{AHI}_{T_{\mathrm{R}}}$ in the STARFM, the changes caused by the atmospheric effect would be treated as land cover changes or noise when generating the aforementioned weightings of 
fine-spatial resolution. Thus, the atmospheric calibration should be considered for the surface reflectance ideally. As the atmospheric correction procedure is usually time-consuming, it prevents us from fully utilizing the AHI 10-min resolution monitoring capability in particular. Therefore, a longer wavelength spectral band (which is much less influenced by the atmospheric scattering and absorption) is utilized for constructing the base of the surface reflectivity. According to the relationships between the visible spectral bands in surface reflectivity, ${ }^{26,27}$ the surface reflectivity of shorter wavelengths can be further obtained. Although the dark target approach is limited over bright surfaces for accurate AOD retrieval due to the variance in spectral reflectivity ratio between dark and bright surfaces, the linear relationship of surface reflectivity between visible and SWIR bands is evidenced in terms of land cover type based on the remotely sensed data after atmospheric correction. ${ }^{26}$ Furthermore, the surface signal used in this study is the relative intensity for the purpose of image fusion in weighting generation. Therefore, the linear relationship between spectral reflectivity is applied to all types of land cover in this study.

In this study, we first process the short-wave infrared (SWIR) spectral band of Landsat-8 OLI central at the spectrum of $1608.5 \mathrm{~nm}$ as the reference of surface reflectance after the systematic calibration with Himawari-8 AHI SWIR spectral band. Then the spatial distribution of SWIR surface reflectance is applied to generate the weightings for image fusion procedure with the diurnal variation from Himawari-8 AHI SWIR. The generated final weightings of SWIR associated with the TOA reflectance in the green band at $T_{1}\left(\mathrm{AHI}_{T_{1}}\right)$ are applied to Eq. (2) to fuse the fine image in the green band which containing the atmospheric effect.

The workflow of proposed STARFM-AP approach is demonstrated in Fig. 3. The main difference between the STARFM and STARFM-AP is the method of surface reflectivity generated with and without SWIR spectral band. Taking the characteristic of the less scattering effect in longer wavelength spectral bands (SWIR), the atmospheric correction of the surface reflectivity in shorter wavelength spectral bands can not only be straightforward but also benefit further atmospheric applications of the fused image, such as the AOD retrieval introduced in the following section.

\subsection{Diurnal AOD Retrieval and Evaluation}

There are generally two kinds of approaches for the AOD retrieval from satellite images, dark target approach ${ }^{28}$ and contrast reduction method. ${ }^{29}$ Considering the blurring effect of the scattering and absorption of atmospheric aerosols (see also the scheme in Fig. 4), contrast reduction method is employed to retrieve the diurnal AOD with the Himawari-8 AHI fused image in this study. It is worthy to notice that the contrast between the bright and dark pixels can be enhanced with the higher spatial resolution after image fusion, which is the main reason that the contrast reduction method is favored on AOD retrieval in this study.

Based on the radiative transfer equation, the satellite observation in Eq. (3) can be further derived as Eq. (4) $)^{30,31}$ under the assumption of the surface reflectance and atmospheric transmittance did not change at time $t_{1}$ and $t_{2}$

$$
\rho^{*}=\rho \frac{T\left(\vartheta_{s}\right) t_{\mathrm{dir}}\left(\vartheta_{v}\right)}{1-\rho_{e} S}+\rho_{e} \frac{T\left(\vartheta_{s}\right) t_{\mathrm{diff}}\left(\vartheta_{v}\right)}{1-\rho_{e} S}+\rho_{\alpha}
$$

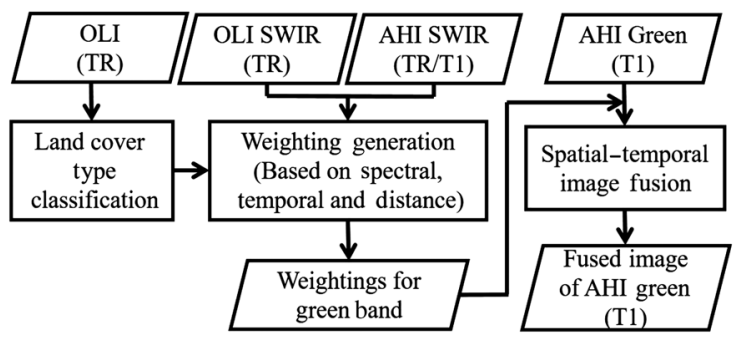

Fig. 3 The flowchart of the STARFM-AP approach. 


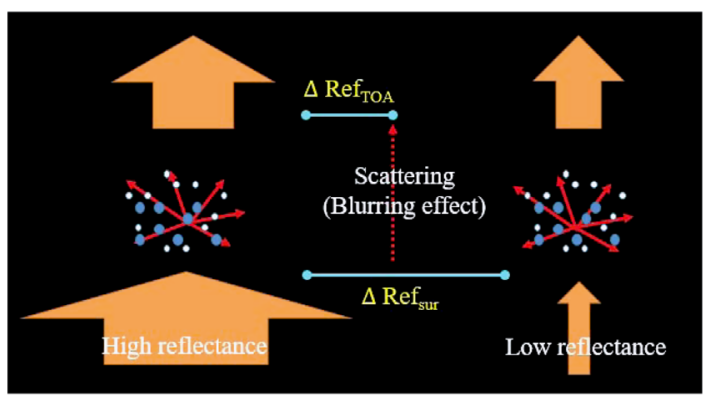

Fig. 4 The concept of contrast reduction (blurring effect).

where $\rho^{*}$ is the apparent reflectance, $\rho_{\alpha}$ is the atmospheric reflectance, $\rho$ is the surface reflectance of the target, $\rho_{e}$ is the average reflectance of the environment, $t_{\mathrm{dir}}$ and $t_{\text {scat }}$ are the direct and the scattered transmission factors, $T\left(\vartheta_{s}\right)$ is the atmospheric transmission factor for the downwelling path, $\vartheta_{s}$ and $\vartheta_{v}$ are the solar and observational zenithal angles, and $S$ is the spherical albedo

$$
\tau_{2}-\tau_{1}=\cos \vartheta_{v} \cdot \ln \left(\frac{\frac{\sigma_{1}\left(\rho^{*}\right)}{\bar{\rho}_{1}}}{\frac{\sigma_{2}\left(\rho^{*}\right)}{\bar{\rho}_{2}}}\right),
$$

where $\tau$ is aerosol optical depth (AOD), $\sigma$ is the standard deviation, $\bar{\rho}$ is the normalization factor, $\vartheta_{v}$ is the sensor viewing angle, and the suffixes 1 and 2 represent the reference time and the target time. The AOD retrievals from Eq. (4) based on fused images $\left(\mathrm{AHI}_{T_{1}}\right)$ will be compared with in situ measurements from AERONET for assessing the performance of the image fusion proposed in this study.

To evaluate the results of the image fusion from the STARFM and STARFM-AP procedures, the direct comparisons between the fused images with the observed images (Landsat OLI) are conducted. In terms of comparing the TOA reflectance differences, the results in preserving atmospheric properties can be identified. For the assessment of further application to the retrieval of diurnal AODs, the coincident measurements of AOD observed from AERONET are also compared.

\section{Results of STARFM and STARFM-AP}

\subsection{Image Fusion}

Figure 5 shows the preliminary results of the fused and original $\mathrm{AHI}_{T_{1}}$ images over the Zhongli District subset at 1100, 1200, 1300, and $1400 \mathrm{~h}$ (local time) on November 16, 2015, respectively. It looks as though the differences of the fused images between the STARFM and STARFM-AP procedures are not obvious from this scale of view. Thus visual comparison from a more zoom-in scale and quantified analyses are carried out in the following section.

\subsection{Validation of Fused Images}

\subsubsection{TOA reflectance comparisons}

The cloud-free and clear sky image datasets acquired on September 13, 2015 were examined first. In terms of comparing the discrepancy in the TOA reflectance, the efficiency in preserving the atmospheric properties can be assessed as well. The ground measurements of the AOD from the AERONET NCU_Taiwan site on September 13, 2015 is 0.101 (clear like) in the green band. The fusion results from the STARFM and STARFM-AP models are shown in Fig. 6, where the reference images are on November 16, 2015. In comparison with the observed image of Landsat-8 OLI on September 13, 2015 [Fig. 6(a)], both the STARFM and STARFM-AP can 


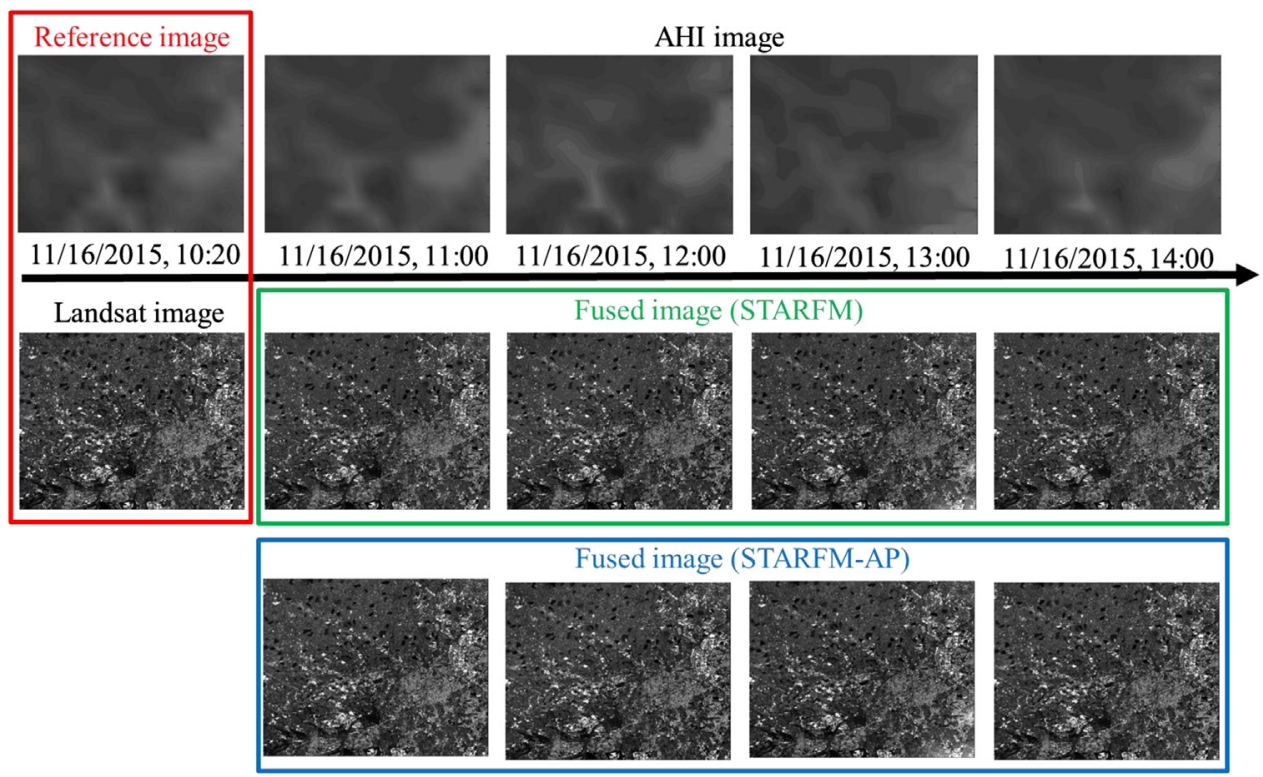

Fig. 5 The results of the fused images with STARFM and STARFM-AP at 1100, 1200, 1300, and $1400 \mathrm{~h}$ (local time) on November 16, 2015.

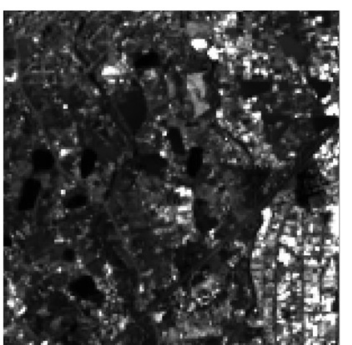

(a)

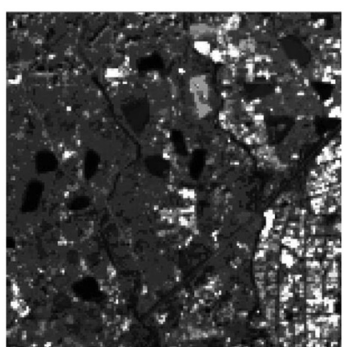

(b)

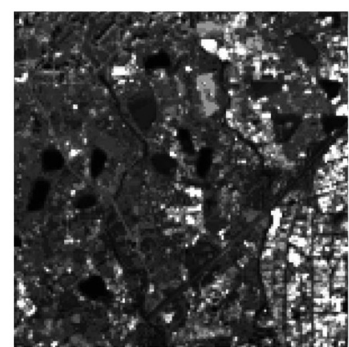

(c)

Fig. 6 Example of the comparison in TOA reflectance between (a) observed image of Landsat-8 OLI image and (b) the fused image by the STARFM procedure from Himawari-8 AHI in $30 \mathrm{~m}$ of spatial resolution, and (c) the same as (b), but processed by STARFM-AP of the image on September 13, 2015 at $1020 \mathrm{~h}$.

provide satisfying fused images where the average absolute differences are 0.1522 [Fig. 6(b)] and 0.1446 [Fig. 6(c)] in reflectance, respectively. Similar findings were reported by Gao et al. ${ }^{16}$

The scatter plots of the TOA reflectance in comparing the fused images between STARFM and STARFM-AP with the observed image are also displayed in Fig. 7 from the results on October 15, 2015. The reference images are the same as the previous cases (i.e., on November 16, 2015). The obvious difference between the STARFM and STARFM-AP fused images happens at around the value 1600 of the TOA reflectance [Fig. 7(b)], where the STARFM images show some systematic error [Fig. 7(a)]. As the STARFM directly applies the green band (more scattering effect than SWIR), the atmospheric effect could reduce down the surface reflectance with regards to the situation of the land cover change. Thus the image fusion process would tend to refer to the reflectance of the neighborhood pixels that have higher reflectivity. As a result, the STARFM fused images show some homogeneous patterns [horizontal strips in Fig. 7(a)] in the local regions. Furthermore, the regression of scatter plots in slope and offset also indicate the better results of STARFM-AP (0.87 and 267.61) than the STARFM (0.82 and 339.08) in producing high-spatial resolution TOA reflectance, which would benefit the AOD retrieval. 


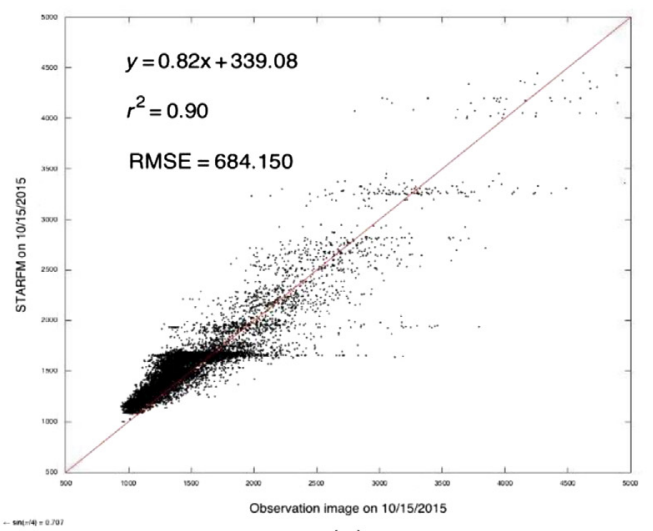

(a)

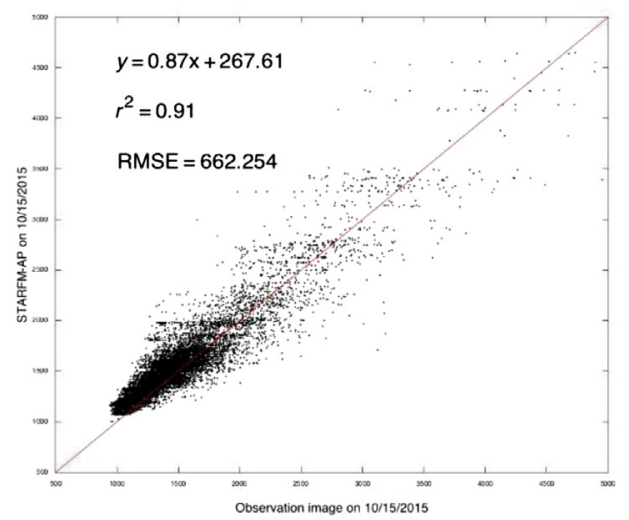

(b)

Fig. 7 The comparisons of the fused images (Himawari-8 AHI) in TOA reflectance between (a) STARFM and (b) STARFM-AP with the observed image (Landsat-8 OLI) on October 15, 2015. The reference images are on November 16, 2015. The value of the reflectance has been normalized from 0 to 10,000 for more clarity.

\subsubsection{Evaluation of $A O D$ retrieval}

In this study, the contract reduction method in terms of dispersion coefficient (see also Sec. 3.3) is applied to retrieve the AOD from the Himawari-8 images and the fused images produced in this study from 8 am to $2 \mathrm{pm}$ in 10-min steps on seven dates (October 25, 2015, October 27, 2015, November 15, 2015, November 16, 2015, November 27, 2015, December 13, 2015, and December 19, 2015). The green band was employed in the comparison due to its better sensitivity to aerosol effects. Theoretically, the window size is one of the variables in AOD calculation using the contrast reduction method [Eq. (4)], which is related to the spatial resolution of satellite image, reflectance of land cover type within the window, and the atmospheric condition. The optimal size of the window for AOD calculation might be different, especially for the different dataset. For fair comparison, the performance on each date was examined with window size from $3 \times 3$ pixels to $21 \times 21$ pixels since the optimal window size for the datasets on each date could be different. In addition, to avoid the possible influence of environmental noises, we filtered out the maximum $20 \%$ and minimum $20 \%$ of the reflectance ${ }^{32}$ for the AOD retrieval and included it in the comparison (denoted by "-20\%" in the figures) as well.

The results of the AOD retrieval from Himawari-8 images before and after fusion on each date are displayed in Fig. 8 for the comparisons. The in situ measurements of AOD from AERONET site are also included as the reference. For the effect of image fusion on AOD retrieval, the performance of STARFM (green circles) and STARFM-AP (blue triangles) are generally much better and stable than those of the original Himawari-8 data (black squares) after comparing with AOD measurements of AERONET on NCU_Taiwan site (red diamonds). The results indicate that the contrast between TOA reflectance can be potentially intensified with higher spatial resolution image for the AOD retrieval based on blurring effect. As the special case with heavier aerosol loading on October 27, 2015 (0.4 to 0.9 in AOD), the original Himawari-8 AHI images won with the best performance as shown in Fig. 8(b). The possible reason is that the AOD observed by an original Himawari-8 pixel will cover a lot of fine pixels after image fusion, which "dilutes" the atmospheric effect when retrieving a local region.

For the effect of atmospheric scattering and absorption (extinction) preservation on AOD retrieval, most of the cases suggest the results produced by STARFM-AP (blue triangles) compared to the counterparts of STARFM (green circles), although the root-mean-square error (RMSE) is similar. In addition to the magnitude, the stability of AOD retrieved from the proposed STARFM-AP approach $(20.7 \%$ in relative error) is also more stable than STARFM (24.4\%), such as the case on November 15, 2015 [Fig. 8(c)].

To provide a quantitative analysis, the AODs retrieved from the Himawari-8, STARFM, and STARFM-AP images were compared with AERONET measurements to calculate the RMSEs and relative errors, which are shown in Table 3. The gray region indicates that those time periods 


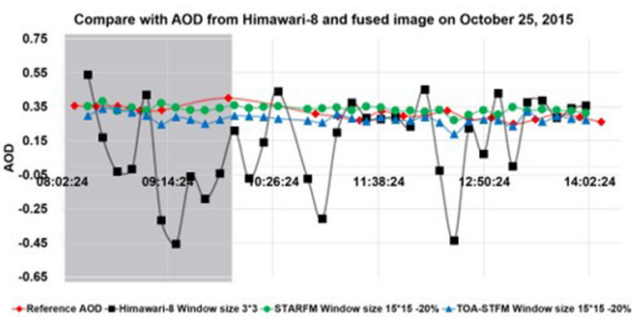

(a)

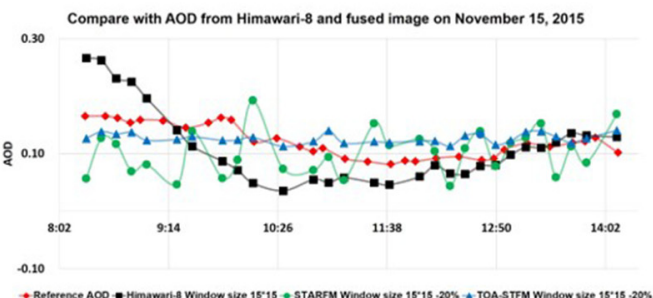

(c)

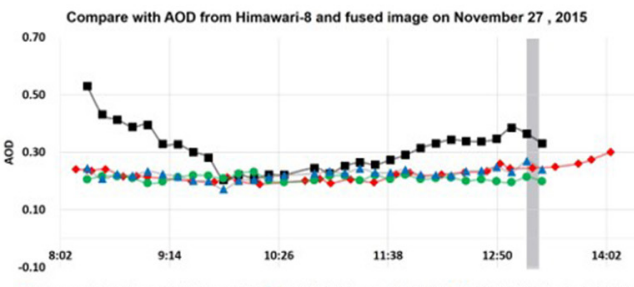

(e)

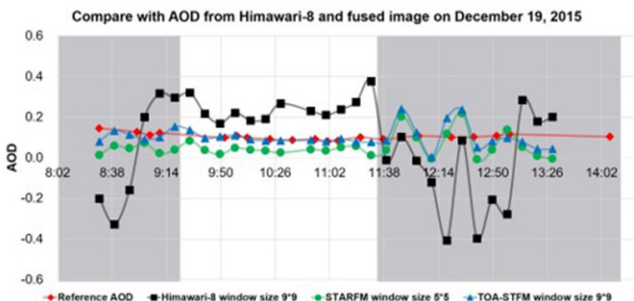

(g)

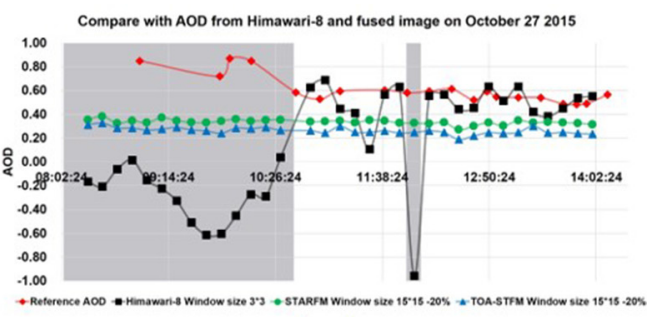

(b)

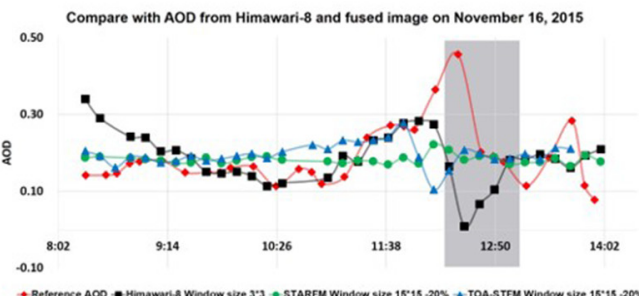

(d)

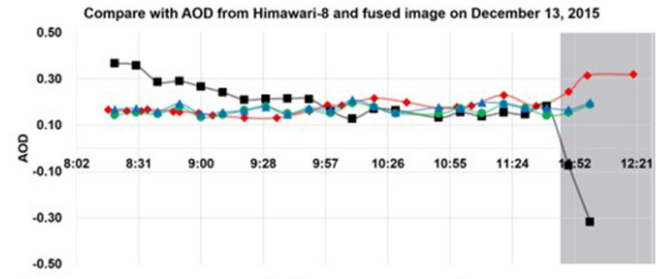

(f)

Fig. 8 The comparisons of the AOD retrievals using the Himawari-8 AHI image (black squares) and the STARFM (green circles) and STARFM-AP (blue triangles) fused image with ground-based measurement (red diamonds) on (a) October 25, 2015, (b) October 27, 2015, (c) November 15, 2015, (d) November 16, 2015, (e) November 27, 2015, (f) December 13, 2015, and (g) December 19, 2015. Gray regions indicate the time periods that have cloud and shadow effects (visually identified).

have cloud and shadow effects (visually identified), which were excluded in the statistical analyses. The averaged relative error of retrieved AODs from original Himawari-8 data, STARFM, and STARFM-AP fused images compared to in situ measurements of AERONET site are 43.7\%, $24.4 \%$, and $20.7 \%$, respectively, as Table 3 summarized. Overall, the better performance of the proposed STARFM-AP in retrieving AODs is indicated in most of the cases, implying that the procedure of atmospheric effect preservation in STARFM-AP for retrieving the AOD in a highspatial resolution is recommended.

\section{Discussions}

\subsection{TOA Reflectance Fusion with SWIR Spectrum}

Surface bidirectional reflectance distribution and atmospheric effect of extinction are the primary factors related to TOA reflectance observed from a satellite sensor. Bidirectional reflectance can 
Table 3 The comparisons of the AOD retrieved from the Himawari-8 AHI image and the STARFM and STARFM-AP fused image in RMSE and relative error.

\begin{tabular}{lcccc}
\hline \hline & $\begin{array}{c}\text { Range of } \\
\text { AOD }\end{array}$ & $\begin{array}{c}\text { Himawari-8 (1000 m) } \\
\text { RMSE/relative error (\%) }\end{array}$ & $\begin{array}{c}\text { STARFM (30 m) } \\
\text { RMSE/relative } \\
\text { error (\%) }\end{array}$ & $\begin{array}{c}\text { STARFM-AP (30 m) } \\
\text { RMSE/relative } \\
\text { error (\%) }\end{array}$ \\
\hline October 25, $2015(32)$ & 0.2 to 0.4 & $0.159 / 46.3$ & $0.048 / 14.3$ & $\mathbf{0 . 0 4 4 / 1 2 . 2}$ \\
October 27, $2015(32)$ & 0.4 to 0.9 & $\mathbf{0 . 0 6 1 / 0 9 . 0}$ & $0.221 / 39.3$ & $0.295 / 53.0$ \\
November 15, $2015(29)$ & 0.1 to 0.2 & $0.033 / 25.5$ & $0.059 / 39.7$ & $\mathbf{0 . 0 2 9 / 2 3 . 3}$ \\
November 16, $2015(32)$ & 0.1 to 0.5 & $0.077 / 21.4$ & $0.071 / 24.7$ & $\mathbf{0 . 0 6 6 / 2 2 . 0}$ \\
November 27, $2015(30)$ & 0.1 to 0.3 & $0.095 / 35.9$ & $\mathbf{0 . 0 2 6 / 0 9 . 5}$ & $0.031 / 11.7$ \\
December 13, $2015(22)$ & 0.1 to 0.3 & $0.034 / 16.3$ & $0.026 / 13.2$ & $\mathbf{0 . 0 2 3 / 1 0 . 8}$ \\
December 19, $2015(30)$ & 0.1 to 0.2 & $0.197 / 151.5$ & $0.069 / 30.1$ & $\mathbf{0 . 0 2 2 / 1 2 . 1}$ \\
Overall result & & $0.091 / 43.7$ & $0.074 / 24.4$ & $\mathbf{0 . 0 7 3 / 2 0 . 7}$ \\
\hline \hline
\end{tabular}

Note: Bold numbers indicate the best results in AOD retrievals.

be taken with the empirical reflectivity in diurnal from a geostationary satellite, whereas the atmospheric effect is mainly fluctuated by the scattering and absorption (extinction) of atmospheric aerosols in visible spectral bands. Instead of the visible band itself, the SWIR spectrum insensitive to atmospheric extinction is proposed to eliminate the atmospheric effect on surface reflectance for image fusion (green band in this study). The better results based on SWIR surface reflectance of image fusion in TOA reflectance [Fig. 7(b)] are evident compared to those of green bands [Fig. 7(a)], either in the scale or offset. In addition, the temporal variance in atmospheric effect is much reduced with the SWIR spectrum for the image fusion in time domain.

\subsection{Improvement in $A O D$ Retrieval}

The characteristics of air pollutants/aerosols in spatial and temporal distribution are obvious, such as urban and rural areas associated with normal and heavy traffic periods. To offer sufficient data, it thus becomes essential to monitor the air quality. Taking the advantages of geostationary- and polar-orbiting satellites in temporal and spatial resolutions, the high-spatiotemporal images are produced with atmospheric information contained in the visible band for aerosol retrieval. Based on the concept of contrast reduction between bright and dark objects caused from atmospheric extinction, the optical depth of suspended aerosols can be derived. Theoretically, the stronger signal in contrast from a higher spatial resolution image facilitates a more accurate AOD retrieval, see also the results before (Himawari-8) and after (STARMF/STARMF-AP) fusion in Table 3. On the other hand, the atmospheric effect/information can be appropriately retained within fused images of the visible band based on SWIR TOA reflectance. The further examinations of fused images in aerosol AOD retrieval with SWIR reflectance exhibit more accurate AOD retrievals (STARFM-AP). The results in Table 3 also confirm the advantages of using SWIR in preserving atmospheric information in visible bands and reducing the fluctuation of atmospheric effect in time domain for the further applications of fused visible images.

\section{Conclusions and Future Work}

Considering the bidirectional reflectance and atmospheric effect, the procedure of the image fusion has been examined with polar and geostationary satellites for high-spatial and temporal AOD retrieval in this study. The high-spatiotemporal resolution image of TOA reflectance is successfully fused with the proposed STARFM-AP approach from Himawari-8 AHI and Landsat- 8 OLI images. The results of the case study and validations demonstrate 
improvements in both TOA reflectance fusion and AOD retrieval. The achievements of this study can be summarized as the integration of radiative characteristics in visible and SWIR spectra to (1) prevent atmospheric effect on surface reflectance for image fusion of TOA reflectance and (2) preserve atmospheric information in fused TOA reflectance for the improvement in AOD retrieval. Therefore, the potential contribution to providing high-spatiotemporal AOD distribution can be expected in monitoring not only diurnal air quality but also the source regions and pathways of pollutants, with the fused images from sequential observations of geostationary satellite.

Nevertheless, we find that the fused images could have a larger reflectance than the actual Landsat OLI images, which could be caused by the blurring effect (aerosol loading). Therefore, to understand the blurring effect in the different spatial resolutions related to optimal window size for AOD retrieval, and include that in the STARFM-AP, should be the future work of this study.

\section{Acknowledgments}

The authors deeply appreciate United States Geological Survey (USGS) and Japan Meteorological Agency (JMA) for providing Landsat and Himawari-8 satellite, and the in situ measurement of AOD from AERONET supported by the National Aeronautics and Space Administration. They are also very grateful to the editor and reviewers for their careful and constructive comments, which have improved the manuscript substantially. The authors would also like to express appreciation to the editorial board and editorial staff. Sincerest thanks for all the expertise and hard work invested in this paper.

\section{References}

1. M. Vittek et al., "Land cover change monitoring using Landsat MSS/TM satellite image data over West Africa between 1975 and 1990," Remote Sens. 6(1), 658-676 (2014).

2. M. C. Hansen et al., "Global land cover classification at $1 \mathrm{~km}$ spatial resolution using a classification tree approach," Int. J. Remote Sens. 21(6-7), 1331-1364 (2000).

3. S. Platnick et al., "The MODIS cloud products: algorithms and examples from terra," IEEE Trans. Geosci. Remote Sens. 41(2), 459-473 (2003).

4. C. Jurgens, "The modified normalized difference vegetation index (mNDVI) a new index to determine frost damages in agriculture based on Landsat TM data," Int. J. Remote Sens. 18(17), 3583-3594 (1997).

5. J. Wang et al., "Improved algorithm for MODIS satellite retrievals of aerosol optical thickness over land in dusty atmosphere: implications for air quality monitoring in China," Remote Sens. Environ. 114(11), 2575-2583 (2010).

6. J. Schwartz et al., "Is daily mortality associated specifically with fine particles?," J. Air Waste Manage. Assoc. 46, 927-939 (1996).

7. S. L. Zeger et al., "Exposure measurement error in time-series studies of air pollution: concepts and consequences," Environ. Health Perspect. 108(5), 419-426 (2000).

8. P. Owili et al., "The associations between types of ambient PM2.5 and under-five and maternal mortality in Africa," Int. J. Environ. Res. Public Health 14, 359 (2017).

9. P. Gupta et al., "Satellite remote sensing of particulate matter and air quality assessment over global cities," Atmos. Environ. 40(30), 5880-5892 (2006).

10. J. Wang and S. A. Christopher, "Intercomparison between satellite derived aerosol optical thickness and PM2. 5 mass: implications for air quality studies," Geophys. Res. Lett. 30(21), 2095 (2003).

11. D. Chu et al., "Global monitoring of air pollution over land from the earth observing system terra moderate resolution imaging spectroradiometer (MODIS)," J. Geophys. Res. 108(D21), 4661 (2003).

12. A. Van Donkelaar et al., "Estimating ground level PM2.5 using aerosol optical depth determined from satellite remote sensing," J. Geophys. Res. Atmos. 111(D21), D21201 (2006). 
Huang, Ho, and Lin: Improving the image fusion procedure for high-spatiotemporal aerosol optical depth...

13. I. Sano et al., "Suspended particulate matter sampling at an urban AERONET site in Japan, part 2: relationship between column aerosol optical thickness and PM 2.5 concentration," J. Appl. Remote Sens. 4(1), 043504 (2010).

14. R. Brakenridge and E. Anderson, "MODIS-based flood detection, mapping and measurement: the potential for operational hydrological applications," in Transboundary Floods: Reducing Risks Through Flood Management, pp. 1-12, Springer, Dordrecht, Netherlands (2006).

15. T. Imai and R. Yoshida, "Algorithm theoretical basis for Himawari-8 cloud mask product," Meteorol. Satell. Center Tech. Note 61, 1-17 (2016).

16. F. Gao et al., "On the blending of the Landsat and MODIS surface reflectance: predicting daily Landsat surface reflectance," IEEE Trans. Geosci. Remote Sens. 44(8), 2207-2218 (2006).

17. T. Hilker et al., "A new data fusion model for high spatial-and temporal-resolution mapping of forest disturbance based on Landsat and MODIS," Remote Sens. Environ. 113(8), 1613-1627 (2009).

18. X. Zhu et al., "An enhanced spatial and temporal adaptive reflectance fusion model for complex heterogeneous regions," Remote Sens. Environ. 114(11), 2610-2623 (2010).

19. M. Wu et al., "A model for spatial and temporal data fusion," J. Infrared Millim. Waves, 31, 80-84 (2012).

20. W. Zhang et al., "An enhanced spatial and temporal data fusion model for fusing Landsat and MODIS surface reflectance to generate high temporal Landsat-like data," Remote Sens. 5(10), 5346-5368 (2013).

21. K. Hazaymeh and Q. K. Hassan, "Spatiotemporal image-fusion model for enhancing the temporal resolution of Landsat-8 surface reflectance images using MODIS images," J. Appl. Remote Sens. 9(1), 096095 (2015).

22. K. Hazaymeh and Q. K. Hassan, "Fusion of MODIS and Landsat-8 surface temperature images: a new approach," PLoS One 10(3), e0117755. (2015).

23. V. K. Shettigara, "A generalized component substitution technique for spatial enhancement of multispectral images using a higher resolution data set," Photogramm. Eng. Remote Sens. 58(5), 561-567 (1992).

24. B. Holben et al., "AERONET - a federated instrument network and data archive for aerosol characterization," Remote Sens. Environ. 66(1), 1-16 (1998).

25. B. Priyadharshini et al., "Discerning the pre-monsoon urban atmosphere aerosol characteristic and its potential source type remotely sensed by AERONET over the Bengal Gangetic plain," Environ. Sci. Pollut. Res. Int. 25(22), 22163-22179 (2018).

26. Y. J. Kaufman et al., "The MODIS 2.1- $\mu \mathrm{m}$ channel-correlation with visible reflectance for use in remote sensing of aerosol," IEEE Trans. Geosci. Remote Sens. 35(5), 1286-1298 (1997).

27. R. C. Levy et al., "The collection 6 MODIS aerosol products over land and ocean," Atmos. Meas. Tech. 6(11), 2989-3034 (2013).

28. P. M. Teillet and G. Fedosejevs, "On the dark target approach to atmospheric correction of remotely sensed data," Can. J. Remote Sens. 21(4), 374-387 (1995).

29. D. Tanré et al., "Estimation of Saharan aerosol optical thickness from blurring effects in Thematic Mapper data," J. Geophys. Res. Atmos. 93(D12), 15955-15964 (1988).

30. N. Sifakis and P. Y. Deschamps, "Mapping of air pollution using SPOT satellite data," Photogramm. Eng. Remote Sens. 5, 1433-1437 (1992).

31. N. Sifakis et al., "Quantitative mapping of air pollution density using Earth observations: a new processing method and application to an urban area," Int. J. Remote Sens. 19, 3289-3300 (1998).

32. N. C. Hsu et al., "Aerosol properties over bright-reflecting source regions," IEEE Trans. Geosci. Remote Sens. 42(3), 557-569 (2004).

Chih-Yuan Huang received his BS and MS degrees in civil engineering and geoinformation engineering from the National Central University in 2007 and 2008, respectively, and his $\mathrm{PhD}$ in geomatics engineering from the University of Calgary in 2014. He is an assistant professor at the Center for Space and Remote Sensing Research, National Central University. He has been the 
Huang, Ho, and Lin: Improving the image fusion procedure for high-spatiotemporal aerosol optical depth...

author of more than 10 journal papers since 2013 and has written three book chapters. His current research interests include GIS, geospatial cyber infrastructure, sensor web, and internet of things.

Hsuan-Chi Ho was a graduate student in the Civil Engineering Department, National Central University. He received his BS degree from the Civil Engineering Department, National United University in 2015, and his MS degree from the Civil Engineering Department, National Central University in 2018. His research mainly focuses on spatiotemporal remote sensing image fusion.

Tang-Huang Lin received his $\mathrm{PhD}$ from the Institute of Space Science, National Central University, Taoyuan, Taiwan, in 2001. He is a professor with the National Central University, since 2006. His research focuses on applying satellite data to retrieve aerosol optical properties for the monitoring of air pollution and atmospheric environment related to the issues of public health, global warming and climate change. 